\title{
The Efficacy of Bacteriophage as a Method of Biofilm Eradication
}

\author{
K TAIT*, L C SKILLMAN and I W SUTHERLAND \\ Institute of Cell and Molecular Biology, Kings Buildings, Edinburgh University, Mayfield Rd, Edinburgh EH9 3JH, UK
}

(Received 11 February 2002; in final form 13 June 2002)

The ability of bacteriophage and their associated polysaccharide depolymerases to control enteric biofilm formation was investigated. Bacteriophages specific for Enterobacter strains were isolated from primary effluent sewage. Combinations of three phages were required before complete eradication of single species biofilms of Enterobacter cloace occurred. Attempts to eliminate a susceptible bacterial population within a dual species biofilm were unsuccessful. It was thought that the structural heterogeneity of the biofilm produced pockets of unattainable, susceptible bacteria. These results suggest that phage and bacteria can co-exist stably within a biofilm. Bacteriophage, would, therefore, make poor tools for the control of biofilm formation. However, the results suggest that combined treatment with bacteriophage polysaccharide depolymerases and disinfectant may provide an alternative control strategy.

Keywords: biofilm; bacteriophage; biocontrol

\section{INTRODUCTION}

With the resistance of pathogenic bacterial strains to traditional antibiotics becoming an increasing problem, there has been interest in the development of alternative methods of control. Bacteriophage and their associated enzymes have been suggested as alternative agents to chemical treatments. This technology was developed, and is still employed within the former Soviet Union, where bacteriophage are reportedly used to treat both primary and nosocomial infections, alone or in conjunction with traditional antibiotics (Holzman, 1998). Nonmedical uses of bacteriophage include the selective removal of pathogenic or problematic species from contaminated surfaces. Kudva et al. (1999) described the isolation of Escherichia coli. O157 antigen-specific bacteriophage. These phages could play a role in biocontrol of $E$. coli in fresh foods, without compromising the viability of other normal flora or food quality. The bacterial spot pathogen of tomato plants, Xanthomonas campestris pv. vesicatoria was successfully controlled with bacteriophage (Flaherty et al., 2000). A viral polysaccharide depolymerase was also evaluated as a potential tool to control fire blight (Kim \& Geider, 2000).

The majority of studies have focused on host: phage dynamics in planktonic cultures, particularly E. coli phages (Bohannan \& Lenski, 2000) and, more recently, marine bacteriophages (SimeNgando, 1997). There has been very little published on the interactions of bacteriophage within biofilms. Bacteriophages are capable of infecting bacterial biofilms. Indeed, a biofilm may be the preferred site for phage reproduction when compared to the less accessible bacteria found in liquid cultures (Wiggins \& Alexander, 1985). Doolittle et al. (1996) examined the infection of E. coli and Pseudomonas aeruginosa biofilms by phage T4 and E27, respectively. Full infection of the thinner E. coli biofilm occurred, but the thicker biofilms of $P$. aeruginosa were only infected at the surface layers; access to the deeper layers was restricted. Hughes et al. (1998a; 1998b) have shown that phage possessing specific polysaccharide depolymerases were able to degrade a pathway through the biofilm EPS to gain access to the bacterial surface. It was shown that the majority of bacteria were being removed from the biofilm due to the action of the enzyme before the cells had a chance to lyse. Adding phage to biofilms containing

*Corresponding author; fax: 01752 633101; e-mail: ktait@pml.ac.uk 
a phage-resistant strain of Enterobacter agglomerans, and again where only soluble enzyme was added to the biofilm, the action of the phage enzyme alone still removed a substantial quantity of biofilm material. It was hypothesised that if the host bacteria produced exopolysaccharide, phages with polysaccharide depolymerase activity could be used to selectively remove the polysaccharide from the biofilm.

The aim of this study was to examine the efficacy of bacteriophage and their polysaccharide depolymerases as a method of biofilm eradication. In addition, the high specificity of phage may make them particularly useful tools in the selective removal of a potentially pathogenic species within a mixed species biofilms. However, in a synergistic dual species biofilm of E. agglomerans and K. pneumoniae, addition of a phage enzyme specific for the EPS of the E. agglomerans strain removed both the strains from the biofilm (Skillman et al., 1999). The inability of a strain-specific phage to eradicate one strain in this mixed species biofilm was thought to be due to the close confinements of the strains within the biofilm. In addition, the interactions between the extracellular polysaccharides produced by the two strains, which resulted in changes to the physical properties of the polymers, may also have affected the selective removal of the E. agglomerans strain. Further studies in the authors' laboratory have indicated that this removal of both species in a dual species biofilm by a strain-specific phage may also have been due to the high titres of phage used. It was indicated that smaller titres of phage might be more successful in selectively removing one species (Napier \& Sutherland, unpublished observations). Smaller titres of bacteriophage were, therefore, used to selectively remove one species from a dual species biofilm. The action of combined treatments of disinfectant and phage enzyme as a potentially effective control strategy was also investigated.

\section{MATERIALS AND METHODS}

\section{Bacteria}

The strains Enterobacter cloace NCTC 5920 and E. agglomerans were used. E. agglomerans strain Ent was isolated from biofilms on industrial surfaces (Dr M V Jones, Unilever Research Laboratory, Port Sunlight, UK).

\section{Bacteriophage Isolation, Purification and Concentration}

Primary effluent sewage was centrifuged at $10000 \mathrm{~g}$ for $20 \mathrm{~min}$ to remove particulate material, then passed through a $0.45 \mu \mathrm{m}$ filter (Millipore) to remove bacteria. The filtered sewage $(50 \mathrm{ml})$ was added to an equal volume of prewarmed double strength YE medium (Sutherland \& Wilkinson, 1965), and $20 \mathrm{ml}$ of overnight bacterial culture added. The optical density $(600 \mathrm{~nm})$ of the culture was monitored over a $12 \mathrm{~h}$ period. A fall in OD due to cell lysis indicated the probable presence of an infectious phage. The culture was centrifuged and the supernatant (containing the phage particles, phage components and the contents of the lysed bacterial cells) was collected. When dialysed, concentrated and filtered (Millipore, $0.45 \mu \mathrm{m}$ pore size), the supernatant acted as an impure source of bacteriophage and bacteriophage proteins (including any polysaccharide depolymerases).

To purify the bacteriophage, the supernatant was serially diluted in phage buffer $(100 \mathrm{mM}$ TRIS, $50 \mathrm{mM} \mathrm{NaCl}, 10 \mathrm{mM} \mathrm{MgSO}_{4} \cdot 7 \mathrm{H}_{2} \mathrm{O}$ ) and $0.1 \mathrm{ml}$ added to $3 \mathrm{ml}$ sloppy agar to which $0.1 \mathrm{ml}$ of overnight culture had been added. The sloppy agar mixture was poured onto a YE agar plate and incubated overnight at $30^{\circ} \mathrm{C}$. Individual plaques were picked off and inoculated into flasks of exponentially growing susceptible bacteria, and again incubated overnight at $30^{\circ} \mathrm{C}$. The above process of centrifugation and filtration was then repeated. The supernatants were concentrated against polyethylene glycol (PEG 6000) for $16 \mathrm{~h}$, filtered (Millipore, $0.45 \mu \mathrm{m}$ pore size) and stored at $4^{\circ} \mathrm{C}$.

\section{Screening of Bacteriophage Activity on Bacteria}

For experimental purposes, a phage was required that would selectively lyse only one species of bacteria in a dual species biofilm. The phages were screened for lytic and polysaccharide depolymerase enzyme activities on strains Ent and 5920. A lawn of each bacterial strain was spotted with $10 \mu \mathrm{l}$ of each concentrated phage solution. The plates were incubated overnight at $30^{\circ} \mathrm{C}$, and the lawns examined for formation of plaques and haloes of decapsulated bacteria, which is indicative of the presence of phage possessing a polysaccharidedegrading enzyme (Lindberg, 1977). The bacteriophage isolated (phage 11229, $\phi$ Ent, Blackburn and Philipstown), and their phage and enzyme activity on the strains Ent and 5920 are shown in Table I. The phage $\phi 1.15$ had previously been isolated (Hughes et al., 1998b).

\section{Biofilm Susceptibility Assays}

The biofilm batch culture vessel contained glass coverslips supported by a stainless steel stand (Hughes et al., 1998a). The stand was capable of supporting $1222 \mathrm{~mm} \times 22 \mathrm{~mm}$ glass coverslips. The vessel held $250 \mathrm{ml}$ of YE medium and was incubated 
TABLE I The bacteriophage isolated and their phage and enzyme activity on Ent and 5920

\begin{tabular}{lccc}
\hline Phage name & Phage activity & Ent & 5920 \\
\hline 11229 & Phage & - & + \\
& Enzyme & - & + \\
$\phi$ Ent & Phage & + & + \\
& Enzyme & + & + \\
$\phi 1.15$ & Phage & - & + \\
\multirow{2}{*}{ Blackburn } & Enzyme & - & + \\
\multirow{2}{*}{ Philipstown } & Phage & - & + \\
& Enzyme & - & + \\
& Phage & + & - \\
\hline
\end{tabular}

at $30^{\circ} \mathrm{C}$. The medium was agitated using a magnetic stirrer (120 rpm).

Single and dual species biofilms were obtained by inoculation of the culture vessel using $24 \mathrm{~h}$ planktonic cultures. The biofilm cultures were incubated $\mathrm{o} / \mathrm{n}(16 \mathrm{~h})$ at $30^{\circ} \mathrm{C}$. Biofilm cell numbers were estimated by viable count. Slides were removed from the culture vessel and washed in 3 changes of sterile phosphate buffered saline (PBS) to remove any unadhered cells. The slide was then placed in a sterile $10 \mathrm{ml}$ beaker containing $1 \mathrm{ml}$ sterile PBS and mechanically crushed to a fine residue to remove the adhered cells. Viable counts were made of the solutions of mechanically removed cells and the number of CFU $\mathrm{cm}^{-2}$ estimated. In the case of the dual species biofilm, the strains Ent and 5920 could be easily distinguished by colony morphology. Three slides were randomly selected from three culture vessels to obtain a mean number of attached cells in the single and dual species biofilm.

For the bacteriophage experiments, single and dual species biofilms were grown on glass coverslips overnight. The slides were removed from the cultures, washed in sterile PBS to remove any unadhered cells and placed into fresh YE medium. The cultures were then inoculated with bacteriophage to allow a 1:10 ratio of phage and cells, and also 1:100 and 1:1000 ratios. Numbers of phage were estimated by titration of the phage solution against a susceptible bacterial strain. In the case of the dual species biofilms, the phage:cell ratios were based on numbers of the susceptible species within the mixed species biofilms. Single and dual species controls without phage were also included. Viable counts were estimated after $24 \mathrm{~h}$. In each case, three slides were randomly selected from three culture vessels to obtain a mean number of attached cells in the single and dual species biofilm.

\section{Separation of Phage Enzyme from Phage Particles}

The phages were produced in high titre by replication in a susceptible host using the methodology of Hughes et al. (1998b). The phage were separated from most bacterial cell debris by centrifugation at $21,000 \mathrm{~g}(8 \times 50 \mathrm{ml}$ tubes $)$ for $20 \mathrm{~min}$. The supernatant was dialysed against phage buffer at $4^{\circ} \mathrm{C}$ for $24 \mathrm{~h}$, and then concentrated against PEG for $16 \mathrm{~h}$. The concentrated supernatant was re-centrifuged at $21,000 \mathrm{~g}$ for $20 \mathrm{~min}$, then passed through Millipore Vivacell 70 membrane filters $(<100 \mathrm{kDa}$ cut-off size) to separate the soluble phage enzyme from the phage particles. The filtrate was refiltered (Millipore, $0.22 \mu \mathrm{m}$ pore size) to ensure no phage remained. The absence of lytic phage was confirmed by spotting on to lawns of susceptible bacteria. The soluble enzyme supernatant was concentrated against PEG and stored at $-20^{\circ} \mathrm{C}$.

\section{Combined Treatments of Phage Enzyme and Disinfectants}

Biofilms of Ent were grown overnight and inoculated with $\phi$ Ent polysaccharide depolymerase. Undiluted disinfectant was added to the biofilms and incubated for $5 \mathrm{~min}$. This was followed by a phage enzyme treatment ( $5 \mathrm{~min})$. Viable counts were then estimated as above.

\section{RESULTS}

\section{Effect of Bacteriophage on Dual Species Biofilms}

Single and dual species biofilms of Ent and 5920 were inoculated with bacteriophage $\phi 1.15$ to allow 1:10, 1:100 and 1:1000 ratios of phage and biofilm bacteria. Similarly, single and dual species biofilms of Ent and 5920 were infected with Philipstown phage. Bacteriophage $\phi 1.15$ has phage and polysaccharide depolymerase activity against strain 5920, but no effect on strain Ent, whereas, Philipstown phage had phage activity against Ent, and no activity against 5920 (Table I). Figure 1 demonstrates the effect of the different concentrations of $\phi 1.15$ phage on single species biofilms of 5920 and dual species biofilms of Ent and 5920, and Figure 2, the effect of Philipstown phage on single species biofilms of Ent and dual species biofilms of the two strains. As $\phi 1.15$ phage had no effect on single species biofilms of Ent, and Philipstown phage had no effect on single species biofilms of 5920, these data are not shown.

An ANOVA test for the three phage treatments of different concentrations, 1:10, 1:100 and 1:1000, on the single species biofilms revealed no significant difference between each treatment (action of $\phi 1.15$ on 5920 single species biofilm $p=0.248$ and for the Philipstown phage infection of Ent single species biofilms, $p=0.46$ ). In the case of the single species biofilms, the three phage treatments produced similar bacterial removal from the biofilms. 


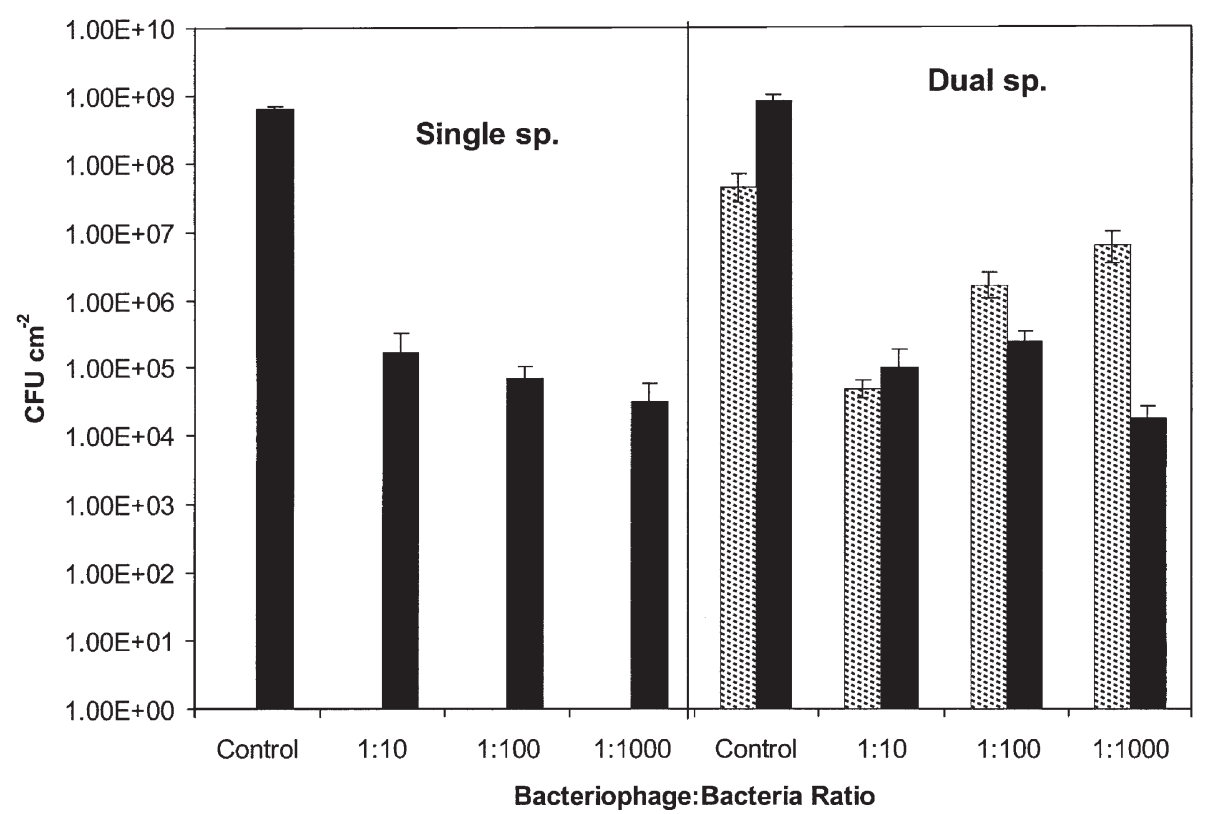

FIGURE 1 The action of $\phi 1.15$ on single species biofilms of 5920 and dual species biofilms of Ent (霝) and 5920 ( $\square$ ). Bacteriophage were inoculated to allow bacteriophage:bacteria ratios of 1:10, 1:100 and 1:1000. Bars $=S E ; n=9$.

This trend was also evident in the dual species biofilms. For both Figure 1 and Figure 2, it can be seen that the numbers of the susceptible bacteria within the dual species biofilms for each phage treatment remained comparatively constant (ANOVA for action of $\phi 1.15$ phage, $p=0.71$ and $p=0.96$ for the Philipstown phage experiments).

Figure 1 also demonstrates that there was an overall decrease in the numbers of Ent, the unsusceptible bacterium with the pair when compared with the dual species control biofilm.
The phage $\phi 1.15$ was an enzyme that is capable of depolymerising the 5920 extracellular polysaccharide (Table I). The action of this enzyme presumably shears off fragments of 5920 polysaccharide, and if Ent and 5920 cells were in close proximity, this would result in removal of both strains from the biofilm. This trend is not as evident with the dual species biofilms infected with Philipstown phage. The dual species biofilms inoculated with 1:100 and 1:1000 ratios of bacteriophage:bacteria contained similar numbers

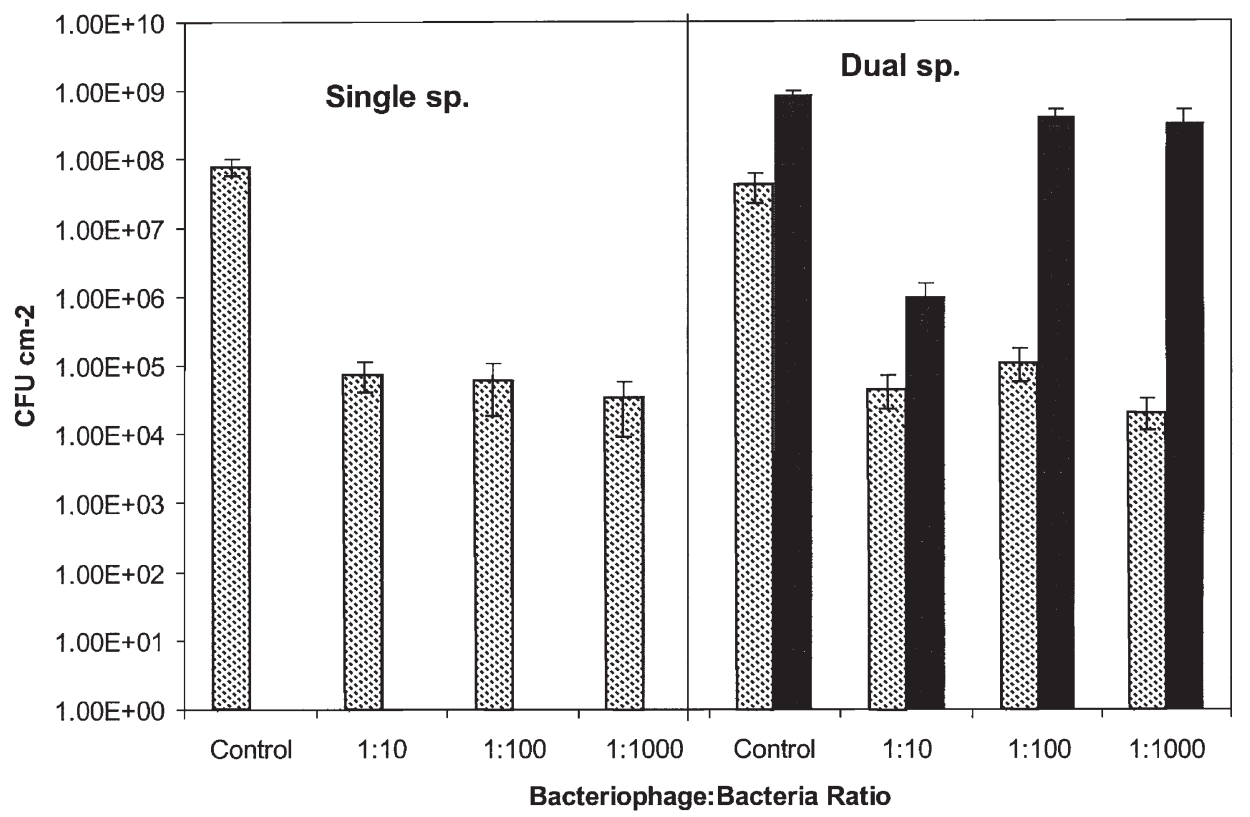

FIGURE 2 The action of Philipstown phage on single species biofilms of Ent and dual species biofilms of Ent (煎) and 5920 ( $\square$ ) Bacteriophage were inoculated to allow bacteriophage:bacteria ratios of 1:10, 1:100 and 1:1000. Bars $=S E ; n=9$. 
of 5920 cells to the control, untreated biofilms. Philipstown phage does not have polysaccharide depolymerase activity (Table I). The action of this phage might be more localised to areas of Ent colonisation within the biofilm, reducing the sloughing off of larger fragments of biofilm. The unsusceptible bacterial population within the dual species biofilms also increased with decreasing phage inoculum size. This was particularly evident with the $\phi 1.15$ phage data. This suggests that the higher phage inocula were more destructive and less selective in their effect on the biofilms.

Most importantly, the results in Figures 1 and 2 indicate that the complete eradication of the single species biofilms and of the susceptible bacterial population in the dual species biofilms was not achieved.

\section{Addition of Multiple Phages to Dual Species Biofilms}

It was hoped that the addition of two or three phages to dual species biofilms of Ent and 5920 would be more successful in selectively removing 5920 cells. From the phage isolated from primary effluent sewage, 11229 and Blackburn bacteriophage had both phage and enzyme activity against 5920, but no activity against Ent. The effect of a combination of phage $\phi 1.15$ and 11229, and of $\phi 1.15,11229$ and Blackburn was, therefore, examined and compared with the action of a single treatment of $\phi 1.15$ phage.

Figure 3 shows the addition of single, double and triple doses of phage to single species biofilms of 5920 and dual species biofilms of Ent and 5920. Biofilms were inoculated with phage to allow a 1:100 ratio of phage and bacteria. The bacteriophage inocula contained equal amounts of each phage. Cultures were incubated for $24 \mathrm{~h}$, and viable counts

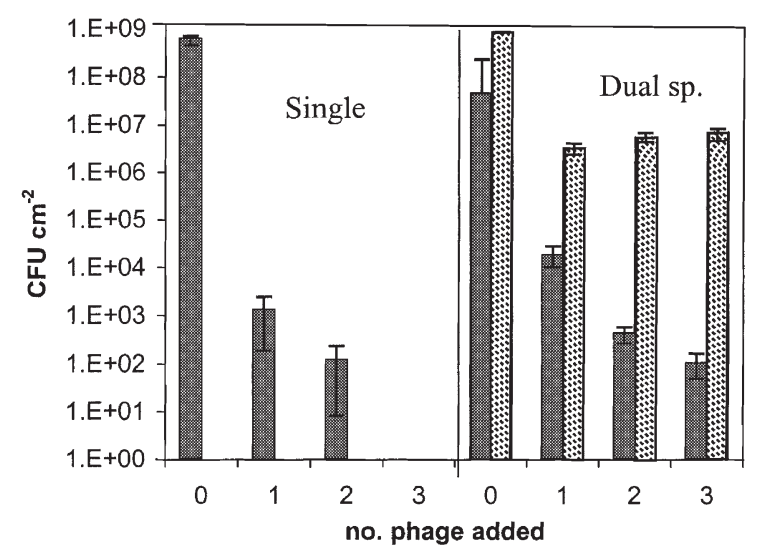

FIGURE 3 Addition of several phages to single species biofilms

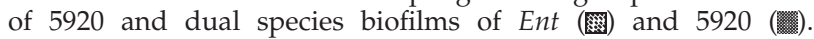
Combinations of $\phi 1.15$ and 11229 (' 2 phage') and $\phi 1.15,11229$ and Blackburn (' 3 phage') were compared to the action of a single dose of $\phi 1.15$. Bars $=S E ; n=9$.

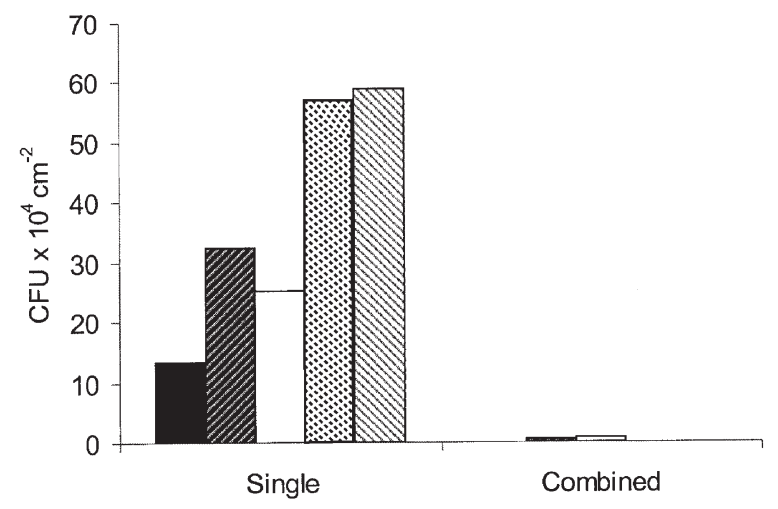

FIGURE 4 Combined treatments of bacteriophage polysaccharide depolymerase and disinfectant. Ent single species biofilms were treated with a hypochlorite based disinfectant $(\mathbb{Z})$, a non-ionic disinfectant $(\square)$, an amphoteric based disinfectant (鿴)

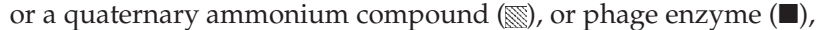
or a combination of the two. All samples were the mean of 3 replicates.

estimated. The results indicate that the inoculum containing three phage types was required to eradicate the single species biofilm of 5920. Although this combination was also most successful in selectively removing 5920 cells from the dual species biofilm, again complete removal of 5920 cells from the biofilms was not achieved.

\section{Combined Effects of Phage and Disinfectant}

The action of combined treatments of disinfectant and phage enzyme as a potentially effective biofilm control strategy was investsigated. Figure 4 demonstrates the removal of single species biofilms of Ent using a polysaccharide depolymerase and a selection of disinfectants, and compares this with combinations of both disinfectant and enzyme. Enzyme was added to the biofilms before the addition of disinfectant, as this was thought to remove cells from the biofilm, giving better access to the remaining attached cells. The combined effects of phage enzyme and disinfectant were more effective than either of these alone.

\section{DISCUSSION}

The complete eradication of both single and dual species biofilms was not achieved. Indeed, communities of bacteriophage and bacteria have been shown to be remarkably stable. Horne (1970) reported the coexistence of phage T4 and E. coli for periods as long as 52 weeks. Much of the coexistence of phage and bacteria has been attributed to the continual appearance of phage-resistance mutants, and phage mutants unable to overcome bacterial 
resistance (Balakshina et al., 1992). Although the emergence of resistance was not evident in this study, the bacteriophage and bacterial biofilms were only incubated for a maximum of $24 \mathrm{~h}$. Given longer periods of incubation, resistance of the bacteria to the bacteriophage would undoubtedly appear. Of more importance to biofilm communities, Schrag and Mittler (1996) provided evidence to suggest that the occurrence of 'spatial refugees', brought about through growth on the walls of culture vessels, might be important in stabilising bacteria-phage interactions. Nutritional limitation is also known to influence stability. Bohannan and Lenski (1997) observed that increasing the input of nutrients led to a large increase in phage numbers, a small increase in bacteria and a reduction in the dynamic stability of both populations. Within a biofilm environment, both spatial heterogeneity and nutritional limitations are common occurrences. These theories suggest growth as a biofilm would increase the stability of bacteria-phage interactions.

Studies by Ripp and Miller (1997) suggest that pseudolysogeny may play an important role in stabilising phage-host interactions. Pseudolysogeny is described as an interaction in which the nucleic acid of the phage resides within a starved host in an unstable, inactive state, allowing the phage to survive for extended periods of time in natural ecosystems. Moebus (1997) provided evidence to suggest the presence of a pseudolysogeny-inducing agent, 'factor $\mathrm{X}$ '. Moebus was able to clot pseudolysogenic cells with an antiphage serum, and proved that this was not due to the presence of virions attached to the cell surface. It was hypothesised that the production of an extracellular 'factor $X$ ' could block the receptors used by phage to infect a bacterium, thus controlling the number of host cells being infected and ensuring the survival of both host and phage. In a densely packed environment such as a bioflim, this strategy would be extremely efficient in stabilising the interactions between phage and host. This theory could also explain why the use of three phages successfully eradicated the single species 5920 biofilm. If each phage used a different receptor, a cell could still be infected with an alternate phage.

In a study of bacteriophage replication in cultures of Staphylococcus epidermidis, Bacillus subtilus and E. coli, cell densities $>10^{4} \mathrm{CFU} \mathrm{ml}{ }^{-1}$ were required before bacteriophage multiplication occurred (Wiggins \& Alexander, 1985). In the present study, the three treatments of different phage concentrations produced similar results in the single species biofilms of Ent and 5920 (Figures 1 and 2). Again, this suggests that bacteriophage are able to detect the number of cells within a population, and control multiplication in order to achieve a stable phage: bacteria coexistence.
The results in this paper indicate that combinations of three phages are required to completely eradicate a single species biofilm of strain 5920 (Figure 3). Biofilms in natural and industrial environments more commonly consist of complex communities of microorganisms. The removal of mixed species biofilms using bacteriophage would become increasingly complex, and it would seem unlikely that bacteriophage are suitable for removing mixed species biofilms from industrial surfaces. A constant fluctuation between phage-resistant and phage-susceptible bacteria, and also changes to phage-host ranges are likely to occur. It has also been suggested that bacteriophage could be used to remove a pathogenic species, such as E. coli O157 from contaminated surfaces (Kudva et al., 1999). These studies reveal that the presence of a nonsusceptible bacterial population within a biofilm could protect bacteriophage-susceptible strains from phage attack, possibly by creating 'spatial refugees' within the depths of the biofilm.

Hughes et al. (1998a) demonstrated that treatment to remove EPS using phage polysaccharide depolymerases was effective in removing large quantities of EPS from surfaces. It was thought that the use of phage enzymes in conjunction with disinfectants could provide an effective means of biofilm removal. Figure 4 demonstrates the increased susceptibility of enzyme-treated biofilms to a disinfectant treatment. It is thought that the polysaccharide depolymerase affords better access for disinfection, and consequently, better removal and eradication. This may be used as a semi-specific treatment in the control of biofilm formation.

\section{Acknowledgements}

$\mathrm{K}$ Tait gratefully acknowledges receipt of a MAFF studentship grant.

\section{References}

Balakshina V V, Kulakov L A, Boronin A M (1992) Dynamics of the interaction of bacteriophage-phi-mf81 with its host, Pseudomonas aeruginosa. Microbiology 61: 741-744

Bohannan B J M, Lenski R E (1997) The effect of resource enrichment on a chemostat community of bacteria and phage. Ecology 78: 2303-2315

Bohannan B J M, Lenski R E (2000) Linking genetic change to community evolution: insights from studies of bacteria and bacteriophage. Ecol Lett 3: 362-377

Doolittle M M, Cooney J J, Caldwell D E (1996) Tracing the interactions of bacteriophage using fluorescent and chromogenic probes. I Ind Microbiol 16: 331-341

Flaherty J E, Jones J B, Harbaugh B K, Somodi G C, Jackson L E (2000) Control of bacterial spot on tomato in the greenhouse and field with h-mutant bacteriophages. Bioscience 35: 882-884

Holzman D (1998) Reassessment of medicinal phage. ASM News 64: $620-622$

Horne M T (1970) Coevolution of Escherichia coli and bacteriophages in chemostat culture. Science 168: 992-993 
Hughes K A, Sutherland I W, Jones M V (1998a) Biofilm susceptibility to bacteriophage attack: the role of phage-borne polysaccharide depolymerase. Microbiology 144 3039-3047

Hughes K A, Sutherland I W, Clark J, Jones M V (1998b) Bacteriophage and associated polysaccharides depolymerasesnovel tools for study of bacterial biofilms. J Appl Microbiol 85 : 583-590

Kim W S, Geider K (2000) Characterisation of a viral EPSdepolymerase, a potential tool for the control of fire blight. Phytopathology 90: 1263-1268

Kudva I T, Jelacic S, Tarr P I, Youderian P, Hovade C G (1999) Biocontrol of Escherichia coli 0157 with 0157-specific bacteriophages. Appl Environ Microbiol 65: 3767-3773

Lindberg A A (1977) Bacterial surface carbohydrates and bacteriophage adsorption. In: Sutherland I W (Ed) Surface Carbohydrates of the Prokaryotic Cell. Academic Press, London, pp 289-356

Moebus M (1997) Investigations of the marine lysogenic bacterium H24. III Growth of bacteria and production of phage under nutrient-limited conditions. Mar Ecol Prog Ser 148: 241-250

Ripp S, Miller R V (1997) The role of pseudolysogeny in bacteriophage-host interactions in a natural freshwater environment. Microbiology 143: 2065-2070

Schrag S J, Mittler J E (1996) Host-parasitic coexistence: the role of spatial refuges in stabilising bacteria-phage interactions. Am Nat 148: $348-377$

Sime Ngando T (1997) Viruses in aquatic ecosystems. A review. Annee Biol 36: 181-210

Skillman L C, Sutherland I W, Jones M V (1999) The role of exopolysaccharides in dual species biofilm development. J Appl Microbiol SS 85: 13-18

Sutherland I W, Wilkinson J F (1965) Depolymerases for bacterial EPS obtained from phage-infected bacteriae. J Gen Microbiol 39: 373-383

Wiggins B A, Alexander M (1985) Minimal bacterial density for bacteriophage replication: implications for significance of bacteriophages in natural ecosystems. Appl Environ Microbiol 49: 19-23 
Copyright of Biofouling is the property of Taylor \& Francis Ltd and its content may not be copied or emailed to multiple sites or posted to a listserv without the copyright holder's express written permission. However, users may print, download, or email articles for individual use. 